\title{
TO THE EQUILIBRIUM MODEL CHOICE IN HETEROGENEOUS AQUEOUS SYSTEMS. 1. THEORETICAL BASIS IN THE CASE OF TWO COEXISTING SOLID PHASES
}

\author{
I. Povar*, Nina Timbaliuc, Tatiana Cazac, \\ Diana Shepel, Oxana Spinu and Ludmila Chiriac \\ Institute of Chemistry, Academy of Sciences of Moldova, 3 Academiei str., Chisinau MD-2028, Republic of Moldova \\ *E-mail: ipovar@yahoo.ca,Phone:+(373)22739736
}

\begin{abstract}
An approach has been developed to determine the equilibrium model in heterogeneous aqueous systems containing a mixture of solid phases from the initial composition of the heterogeneous mixture and the equilibrium values of the $\mathrm{pH}$. This approach allows to significantly reduce the quantity of measured data, properly for solubility measurements. By using the new deduced equations it is possible to determine the solubility products of precipitates on the basis of the known composition of initial mixtures and $\mathrm{pH}$ values of saturated solutions avoiding some difficult experimental solubility measurements. The derived relations are useful for solving both direct and indirect equilibrium problems.
\end{abstract}

Keywords: equilibrium model, heterogeneous system, solubility product, $\mathrm{pH}$ value, solid phase.

\section{Introduction}

The full definition of equilibrium model includes the number, nature and stability of chemical species in considered systems [1-4]. Actually, the equilibrium model refers to a set of equilibrium equations and the associated equilibrium constants. The objective of this study is to develop a new method for choice of equilibrium model characteristics that will allow to significantly reducing the number and volume of measured data.

We will analyze the heterogeneous aqueous systems in which, depending on the medium acidity, various individual solid phases may occur. We will be also taking into account the possibility of coexistence of individual precipitates, especially in the cases of closed values for their product solubility values $p K_{S}=-\log K_{S}$.

There are two main methods to obtain the equilibrium (thermodynamic) constants that characterize the species stability in solid and liquid phases:

a) Solubility method or the method of residual concentrations. The relation between experimental data obtained by these two methods was revealed in papers [5-7]. Accurate measurement of solubility of sparingly-soluble compounds often requires special experimental consideration and approaches. The traditional saturation shakeflask procedures for measuring the equilibrium solubility are manually intensive, time- and sample-consuming, and may be prone to systematic errors not easily recognized, especially when the $\mathrm{pH}$ is not carefully measured, when aggregation or complexation reactions take place (unexpectedly altering spectroscopic properties), or when highly insoluble substances are considered (exceeding the sensitivity of the analytical method) [8].

b) Potentiometric method for determination of all the component activities of the precipitate (or precipitates) in saturated solutions [5-7]. This method allows to examine poorly soluble compounds not only in dilute solutions, but in concentrated solutions and in the presence of other electrolytes as well. Recently a new potentiometric method has been proposed which overcomes some of the limitations of standard approaches [9-13]. The new automated potentiometric method [9] was shown to be reliable for determining the solubility-pH profiles of the uncharged substances. Its speed compared to conventional equilibrium measurements (entire solubility-pH profile in 3-10 hr), its sound theoretical basis [9], its ability to generate the full solubility-pH profile from a single titration, and its dynamic range (estimated to be seven orders of magnitude) make the new $\mathrm{pH}$-metric method an attractive addition to traditional approaches.

The first method is associated with complex measurements of the solubility of compounds (or their residual concentrations), while the second one is limited by a narrow assortment of existing stable and reproducible ion-selective electrodes.

Original methods for the determination of solubility products of poorly soluble hydroxides, acids salts and complexonates, based on the potentiometric measurement of only equilibrium $\mathrm{pH}$ values of heterogeneous systems as functions of the initial concentrations of the precipitate components, which are known when preparing the mixture were developed in [5-7]. The values for the equilibrium constants as well as the equilibrium and residual concentrations calculated for a series of real systems from known experimental data were in good accordance with those reported in literature under the same conditions.

As it will be shown in this paper, our approach for determining species solubilities and equilibrium constants, based 
on new developed equations that require less quantity (and number) of needed experimental data, can be successfully applied as well for the heterogeneous systems containing two coexisting solid phases (precipitates).

\section{Theory, Results and Discussion}

The methods of the potentiometric determination of product solubility $K_{S}$ values for sparingly soluble acids and bases (the systems of type $\boldsymbol{a}$ ) as well for sparingly soluble salts of different compositions, in the case of a single solid phase formation (the systems of type b) (avoiding solubility measurements) were developed in [5-7]. For the systems $\boldsymbol{a}$ and $\boldsymbol{b}$ the equations for calculating $K_{S}$ values and the necessary experimental data are summarized in Table 1.

Possible composition of individual solid phases and their solubility products

Table 1

\begin{tabular}{|l|l|l|}
\hline $\begin{array}{c}\text { Solid phase } \\
\text { composition } \\
\text { and medium }\end{array}$ & \multicolumn{1}{|c|}{ Equations for calculation } & \multicolumn{1}{|c|}{ Needed experimental data } \\
\hline $\begin{array}{l}H A_{(S)} \\
\text { acid solutions }\end{array}$ & $K_{S}=[H]\left(C_{A}^{0}-C_{H}^{0}+[H]\right)$ & $C_{A}^{0}, C_{H}^{0}, p H$ \\
\hline $\begin{array}{l}M A_{2(S)} \\
\text { acid solutions }\end{array}$ & $C_{A}^{0}-C_{H}^{0}-2 C_{M}^{0}+[H]=$ & $C_{A}^{0}, C_{M}^{0}, C_{H}^{0}, p H$ \\
\hline $\begin{array}{l}M H A_{(S)} \\
\text { acid solutions }\end{array}$ & $\left.\begin{array}{l}C_{A}^{0}-C_{H}^{0}=K_{S}^{\prime \prime}[M]^{-1}[H]^{-1}-[H] \\
C_{M}^{0}-C_{H}^{0}=[M]-[H]-K_{S} K[M]^{-1}\end{array}\right)$ & $\begin{array}{l}C_{A}^{0}, C_{M}^{0}, C_{H}^{0}, p H, p M= \\
=-\log [M]\end{array}$ \\
\hline $\begin{array}{l}M(O H)_{2(S)} \\
\text { alkaline } \\
\text { solutions }\end{array}$ & $\begin{array}{l}C_{O H}^{0}-2 C_{M}^{0}=K_{S}[H]^{-2}(\chi-2 \alpha)+K_{W}[H]^{-1} \\
\alpha=\sum_{i=0} K_{i}[H]^{-i}, \quad \chi=\sum_{i=1} i K[H]^{-i}\end{array}$ & $C_{M}^{0}, C_{O H}^{0}, p H$ \\
\hline
\end{tabular}

(Here the indices “ $a$ ”, “ $b$ ” and “s” represent $a c i d$, base and salt respectively).

We will consider comprehensively one of possible cases of coexistence of two precipitates (denoted by the index "s") with a common ion $A$ ":

$$
\begin{aligned}
& H A_{(S)} \Leftrightarrow H+A, \quad K_{S}^{\prime}=[H][A] \\
& M A_{2(S)} \Leftrightarrow M+2 A, \quad K_{S}^{\prime \prime}=[M][A]^{2}
\end{aligned}
$$

Here and underneath the charges of species are omitted for simplicity.

Using well-known methods of accounting for the non-ideality of real systems, it is easy to calculate the thermodynamic constants from the concentration ones [5-7]. In liquid stage, we shall consider a series coexistent equilibrium. For acid solutions we will take into consideration the side reactions of anion $A^{\text {- }}$ protonation:

$$
H A \Leftrightarrow H+A, \quad K=[H][A]
$$

The mass balance conditions in this heterogeneous system are defined as follow [5-7]:

$$
\begin{gathered}
C_{M}^{0}=P^{\prime \prime}+[M] \\
C_{A}^{0}=P^{\prime}+2 P^{\prime \prime}+C_{A}^{r}=P^{\prime}+2 P^{\prime \prime}+[A]+[H A]=P^{\prime}+2 P^{\prime \prime}+[A](1+K[H]) \\
C_{H}^{0}=P^{\prime}+C_{H}^{r}=P^{\prime}+[H]-[O H]+[H A]=P^{\prime}+[H]-K_{W}[H]^{-1}+K[H][A]
\end{gathered}
$$

where $P$ is the amount of the $i$ th ion in the precipitate per 1 unity of the solution volume (expressed in mol/L), $\mathrm{C}_{\mathrm{i}}^{\mathrm{r}}$ is the residual (or analytical) concentration of the component " $i$ " of the precipitate in saturated solutions, i.e. its concentration in the solution after the separation of the solid and liquid phases, mol/L [5,6].

The elimination of $P$ from the set of equations above by the linear combination of the mass balance equations (4)-(6) yields: 


$$
\begin{aligned}
& C_{A}^{0}-C_{H}^{0}-2 C_{M}^{0}=C_{A}^{r}-C_{H}^{r}-2[M]= \\
& =[H A]+[A]-[H]+[O H]-2[M]
\end{aligned}
$$

Taking into account the MAL (mass action law) equations (1) and (2), the equation (7) may be rewritten as follows:

$$
C_{A}^{0}-C_{H}^{0}-2 C_{M}^{0}=K_{S}^{\prime}[H]^{-1}-[H]-2 \frac{[H]^{2} K_{S}^{\prime \prime}}{\left(K_{S}^{\prime}\right)^{2}}
$$

If the composition of initial mixture $\left(C_{i}^{0}\right)$ at the measured equilibrium $\mathrm{pH}$ value of saturated solutions is known, the equation (8) contains 2 variables: $K_{S}^{\prime}$ and $K_{S}$ ". Measuring the $\mathrm{pH}$ values for two different compositions of the initial mixture, we compose a system of two equations with two unknown values $K_{S}$ ' and $K_{S}$ ", which is unproblematic to solve. Furthermore, from the equations (1) and (2) it is possible to calculate the equilibrium concentrations $[M]$ and $[A]$. If the values $K_{S}$ are known apriori, the satisfactory coincidence between calculated results and tabulated data may serve as a proof of the correctitude of the chosen equilibrium model.

If the calculated equilibrium characteristics for a certain equilibrium model keep the constancy of their values within the studied area of the component concentrations, one may affirm that this model is chosen properly.

The other possible compositions of a mixture of solid phases and the equations for their solubility product calculation are summarized in Table 2.

\begin{tabular}{|c|c|c|}
\hline $\begin{array}{c}\text { Solid phase } \\
\text { composition and } \\
\text { medium }\end{array}$ & Equations for calculation & Needed experimental data \\
\hline$H A_{(S)}+M A_{2(S)}$ & $C_{A}^{0}-C_{H}^{0}-2 C_{M}^{0}=$ & $C_{A}^{0}, C_{M}^{0}, C_{H}^{0}, p H$ \\
\hline$\downarrow$ & $=K_{c}^{\prime}[H]^{-1}-[H]-2\left([H]^{2} K_{S}^{\prime \prime} /\right.$ & \\
\hline $\begin{array}{c}K_{S}^{\prime} \quad K_{S}^{\prime \prime} \\
\text { acid solutions }\end{array}$ & $\left.\left(K_{S}^{\prime}\right)^{2}\right)$ & \\
\hline$H A_{(S)}+M H A_{(S)}$ & $K_{S}^{\prime}=[H]\left(C_{A}^{0}-C_{H}^{0}+[H]\right)$ & $C_{A}^{0}, C_{H}^{0}, p H, p M$ \\
\hline$\downarrow \quad \downarrow$ & $K_{S}^{\prime \prime}=K_{S}^{\prime}[M]$ & \\
\hline $\begin{array}{c}K_{S}^{\prime} \quad K_{S}^{\prime \prime} \\
\text { acid solutions }\end{array}$ & & \\
\hline$M A_{2(S)}+M H A_{(S)}$ & $C^{0}-C^{0}=[H]+K_{S}^{\prime} K[H]^{2} \quad\left(K_{S}^{\prime \prime}\right)^{2}$ & $C_{M}^{0}, C_{H}^{0}, p H$ \\
\hline$\downarrow \quad \downarrow$ & $K_{S}^{\prime \prime} \quad K_{S}^{\prime}[H]^{2}$ & \\
\hline $\begin{array}{l}K_{S}^{\prime} \quad K_{S}^{\prime \prime} \\
\text { acid solutions }\end{array}$ & & \\
\hline$M A_{2(S)}+M(O H)_{2(S)}$ & $C_{0}^{0} \quad 1\left[C_{0} \quad\left(K_{S}^{\prime}[H]^{2}\right)^{1 / 2}\right]$ & $C_{A}^{0}, C_{M}^{0}, C_{O H}^{0}=-C_{H}^{0}, p H$ \\
\hline$\downarrow \quad \downarrow$ & $\mathrm{C}_{M}=\overline{2}\left[\mathrm{C}_{A}-\left(\overline{K_{S}^{\prime \prime}}\right)\right]+K_{S}[H] \alpha+$ & \\
\hline $\begin{array}{l}K_{S}^{\prime} \quad K_{S}^{\prime \prime} \\
\text { alkaline solutions }\end{array}$ & $\begin{array}{l}+\frac{1}{2}\left(C_{O H}^{0}-K_{W}[H]^{-1}+[H]-K_{S}^{\prime \prime}[H]^{2} \chi\right) \\
\alpha=\sum_{i=0} K_{i}[H]^{-i}, \quad \chi=\sum_{i=1} i K[H]^{-i}\end{array}$ & \\
\hline
\end{tabular}

Possible composition of a mixture of solid phases and their solubility products

Table 2

For better understanding the quintessence of the developed approach a real heterogeneous system, that can contain jointly two solid phases, will be examined. The heterogeneous system cadmium caprate-saturated solution can 
serve as an example of such system because of the practical significance of polyvalent metal ions in the form of lowsoluble soaps with potassium salts of saturated fatty acids [14]. For this system the following constants of equilibrium are known: $\log K^{\prime}=-10.12$ and $\log K^{\prime \prime}=-8.74$ [15]. By means of experimental measurements the authors [14] proved that the simultaneous coo-precipitation of the cadmium caprate $C d A_{2(S)}$ and caprinic (or n-capric) acid $H A_{(S)}$ takes place in acidic solutions, the hydrolysis of cadmium ion can be neglected. The equilibrium $\mathrm{pH}$ values in the case of coexistence of two solid phases are calculated by the equation (8). After that, using the above combined equations of the mass action law and the mass balance, we can easily calculate the distribution diagrams of the metal ion and anion in the solid and complex species in aqueous solution, i.e., the heterogeneous equilibrium diagrams (depending on the problem to be solved, one can calculate the distribution of species containing one or other component of solid phases) [16].

Initially, we will introduce the following equations for calculating the partial molar fractions $\gamma_{i}$ of species containing the anion $A^{-}$in the heterogeneous system, in which reactions (1)-(3) simultaneously occur:

$$
\gamma_{H A(S)}=\frac{P^{\prime}}{C_{A}^{0}}, \quad \gamma_{M A 2(S)}=\frac{2 P^{\prime \prime}}{C_{A}^{0}}, \quad \gamma_{A^{-}}=\frac{[A]}{C_{A}^{0}}, \quad \gamma_{H A}=\frac{[H A]}{C_{A}^{0}}, \quad \gamma_{S U M}=\frac{C_{A}^{r}}{C_{A}^{0}}=\frac{[A]+[H A]}{C_{A}^{0}}
$$

The subscript "sum" denotes the sum of the molar fractions of all soluble species. The comparison of the equations (9) with equation (5) implies that

$$
\gamma_{H A(S)}+\gamma_{M A 2(S)}+\gamma_{S U M}=\gamma_{H A(S)}+\gamma_{M A 2(S)}+\sum_{i=0} \gamma_{H_{i} A}=1
$$

In their appearance, the heterogeneous chemical equilibrium diagrams (see Fig. 1) resemble the distribution diagrams of complex species in a homogeneous solution, but the molar fractions of species $\gamma_{i}$ also depend on the initial composition of the mixture and, hence, for the system (1)-(3) are functions of three variables: $\gamma_{i}=f\left(C_{A}^{0}, C_{M}^{0}, p H\right)$. From the calculated diagram $\gamma_{i}=f(p H)_{C_{i}^{0}}$, i.e. $C_{A}^{0}=$ const and $C_{M}^{0}=$ const (Fig.1) one can deduct that the solid phases

$C d A_{2(S)}$ and $H A_{(S)}$ coexist within the $\mathrm{pH}$ values 5 to 6 . So, the equation (8) is valid only inside this $\mathrm{pH}$ range. The nature of the formed precipitates can be checked by the IR examination after their isolation from aqueous solution with different $\mathrm{pH}$ values [14].

In consequence, knowing one of the equilibrium constants $K_{S}{ }^{\prime}$ or $K_{S}$ " , we can determine the second one by means of the deduced equation (8). The later equation can also be used in the early stage of developing a equilibrium model for the new compounds.

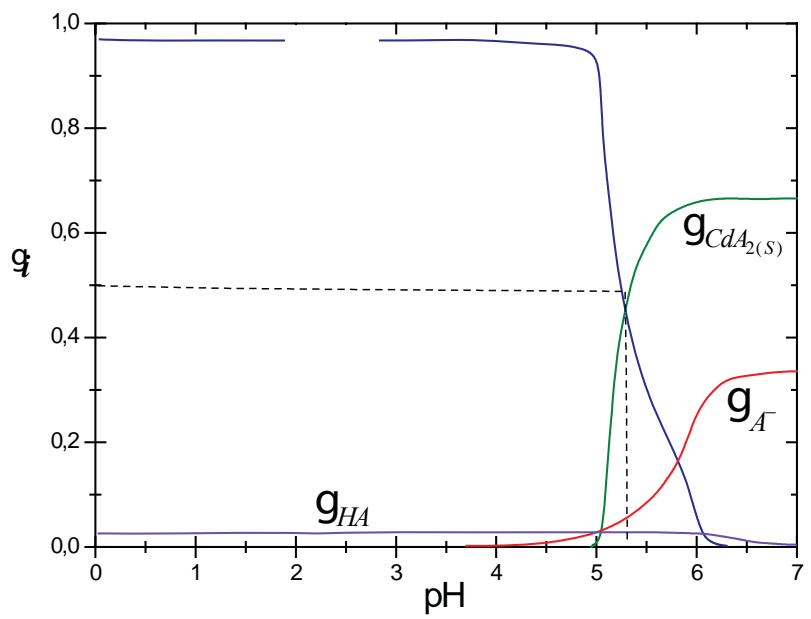

Fig. 1. The distribution of the caprat ion in the heterogeneous system cadmium caprate $C d A_{2(S)}$ - capinic acid $H A_{(S)}$ - aqueous solution as a function of $\mathbf{p H}$ at $C_{C d}^{0}=2.23 \cdot 10^{-3} \mathrm{~mol} / \mathrm{L}$ and $C_{A}^{0}=6.69 \cdot 10^{-3} \mathrm{~mol} / \mathrm{L}$. 


\section{Conclusions}

Therefore, the deduced relations are useful for solving both the direct (I) and indirect (II) equilibrium problems:

(I) Direct problem: calculating the equilibrium composition of heterogeneous mixture using the tabulated constants;

(II) Indirect problem: determining the equilibrium constants (the equilibrium model) on the basis of experimental data.

As it can be seen from the data of Table 2, in many cases the obtained equations allow to determine the values of equilibrium constants, mainly $K_{S}$, using the $\mathrm{pH}$ values of saturated solutions (avoiding this way the traditional, sometimes rather hard, solubility measurements).

The equilibrium constants computed by the developed equations may also produce the "seed" values for the iterative least squares procedure for $\log K_{S}$ refinement, using the existing processing programs.

The developed approach can be applied without difficulty in the case of formation of a mixture of precipitates for arbitrary compositions.

\section{Acknowledgments}

This work was done with the support of the SCOPES 2009-2012 joint research grant.

\section{References}

[1]. POVAR, I. Numerical differentiation of the formation function and selection of a chemical equilibrium model. Russ. J. Inorg. Chem. 1993, 38, 1787 - 1790.

[2]. POVAR, I. Derivative of the formation function in respect to equilibrium ligand concentration in the case of polynuclear complex formation in two-component systems. Russ. J. Inorg. Chem. 1993, 38, 1782 - 1786.

[3]. POVAR, I. Functional transformations of experimental data and the chemical model of equilibrium for polynuclear systems - 1. Family of auxiliary concentration functions curves with common cross-over point. Talanta. 1994, 41, 1363 - 1368.

[4]. POVAR, I. Utilization of some partial derivatives of Olerup function for investigating chemical equilibria in polynuclear systems. Rev. Roum. Chim. 1995, 40, 319 - 324.

[5]. POVAR, I. Potentiometric determination of solubility products of poorly soluble hydroxides and acids. J. Anal. Chem. 1998, 53, 1113 - 1119.

[6]. POVAR, I. Potentiometric determination of solubility products and equilibrium ion concentrations for poorly soluble salts. Russ. J. Gen. Chem. 2000, 70, 501 - 507.

[7]. POVAR, I. Determination of the stability of slightly soluble complexonate from pH metric data. Can. J. Chem. 2001, 79, 1166-1172.

[8]. AVDEEF, A.; BERGER, C.M.; BROWNELL C. pH-metric solubility. 2: Correlation between the acid-base titration and the saturation shake-flask solubility - pH methods. Pharm. Res. 2000, 17, 85-89.

[9]. AVDEEF, A. pH-metric solubility. 1. Solubility-pH profiles from Bjerrum plots. Gibbs buffer and pKa in the solid state. Pharm. Pharmacol. Commun. 1998, 4, 165-178.

[10]. ROSEMAN, T.J.; YALKOWSKY, S.H. Physicochemical properties of prostaglandin $F_{2 \alpha}$ (tromethamine salt): solubility behavior, surface properties, and ionization constants. J. Pharm. Sci. 1973, 62, 1680-1685.

[11]. KAUFMAN, J.J.; SEMO, N.M.; KOSKI, W.S. Microelectrometric titration measurement of the $p K_{a} s$ and partition and drug distribution coefficients of narcotics and narcotic antagonists and their $\mathrm{pH}$ and temperature dependence. J. Med. Chem. 1975, 18, 647-655.

[12]. STRENG, W.H.; ZOGLIO, M.A. Determination of the ionization constants of compounds which precipitate during potentiometric titration using extrapolation techniques. J. Pharm. Sci. 1973, 73, 1410-1414.

[13]. TODD, D.; WINNIKE, R.A. A rapid method for generating pH-solubility profiles for new chemical entities. Abstr. $9^{\text {th }}$ Ann. Mtng., Amer. Assoc. Pharm. Sci., San Diego, 1994.

[14]. POVAR, I.; SAZONOVA, V.F.; SKRYLEVA, T.L.; SKRYLEV, L.D., Thermodynamic prediction and experimental substantiation of conditions of phase transformations in the system cadmium caprate-saturated aqueous solution. Russ. J. Gen. Chem. 1999, 69, 1698 - 1701.

[15]. KUMOK, V.N.; KULESHOVA O.M.; KARABIN L.A. Solubility products. 1983, Novosibirsk: Nauka, 266 p (in Russ).

[16]. POVAR, I. Method for graphic representation of heterogeneous chemical equilibria in systems sparingly soluble compound - complexing agent - aqueous solution. Russ. J.Inorg. Chem. 1997, 42, 607 - 612. 